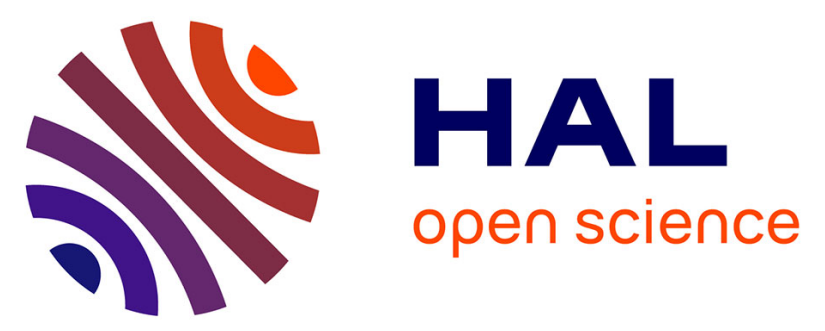

\title{
New versatile and linear optical sensor based on electro-optical modulation and compensation
}

Rémy Claverie, Jean-Paul Salvestrini, Marc Fontana, Pascal Ney

\section{To cite this version:}

Rémy Claverie, Jean-Paul Salvestrini, Marc Fontana, Pascal Ney. New versatile and linear optical sensor based on electro-optical modulation and compensation. Review of Scientific Instruments, 2008, 79, pp.123103. 10.1063/1.3036979 . hal-00302942

\section{HAL Id: hal-00302942 \\ https://hal.science/hal-00302942}

Submitted on 1 Dec 2021

HAL is a multi-disciplinary open access archive for the deposit and dissemination of scientific research documents, whether they are published or not. The documents may come from teaching and research institutions in France or abroad, or from public or private research centers.
L'archive ouverte pluridisciplinaire HAL, est destinée au dépôt et à la diffusion de documents scientifiques de niveau recherche, publiés ou non, émanant des établissements d'enseignement et de recherche français ou étrangers, des laboratoires publics ou privés. 


\title{
New versatile and linear optical sensor based on electro-optical modulation and compensation
}

\author{
Rémy Claverie, Jean Paul Salvestrini, ${ }^{\text {a) }}$ Marc D. Fontana, and Pascal Ney \\ Laboratoire Matériaux Optiques, Photonique et Systèmes, University of Metz, Supélec, UMR CNRS 7132, 2, \\ Rue E. Belin, Metz 57070, France
}

(Received 13 August 2008; accepted 3 November 2008; published online 1 December 2008)

\begin{abstract}
This paper is devoted to the description and characterization of a new electro-optic sensor based on a polarimetric device operating in reflection mode, and on a closed-loop controller. The main features of the proposed sensor are versatility, insensitivity to the laser beam optical power fluctuation, and fully linear working mode. The resolution of the sensor, in terms of phase shift, is shown to be better than $\pm 20 \mathrm{mrad}$ and its accuracy to $\pm 50 \mathrm{mrad}$. (C) 2008 American Institute of Physics. [DOI: 10.1063/1.3036979]
\end{abstract}

\section{INTRODUCTION}

In general, optical sensors have many advantages over conventional electronic sensors. These include immunity to electromagnetic interference, avoidance of ground loops, high sensitivity, large bandwidth, capability of remote operation, small size, and light weight. ${ }^{1}$ Optical sensors have been widely investigated to measure almost every aspect of physical parameters, chemical agents, and biological species, especially in harsh environments, where extreme physical conditions often prevent conventional electrical sensors from being used or make them difficult to use. The use of polarimetry for the sensing of temperature, ${ }^{2}$ electric field, ${ }_{7}^{3,4}$ surface charge distribution, ${ }^{5}$ stress and strain, ${ }^{6}$ humidity, ${ }^{7}$ and in the detection of analyte concentrations ${ }^{8}$ has existed for several years. This technique relies on the refractive index sensitivity of crystals to many physical external parameters leading to the modification of the polarization state of a light-beam going across them. In the simplest optical polarimetric sensor, an optical sensing device is located between an optical polarizer and a polarization analyzer. The sensing device splits the incident lightwave into two orthogonal linearly polarized waves. These two waves propagate at different velocities in the polarizing device (because of the birefringence). Their relative phase are then shifted by a quantity $\Gamma$. After being analyzed, by a polarization analyzer, the emerging optical intensity is a function of the phase shift $\Gamma$, which can be a function of various physical parameters. Thus, the value of the physical parameter is quantified by the measurement of the optical intensity, and its variation according to the change in the physical parameter, at the output of the arrangement. To improve the sensitivity and the accuracy of such an optical sensor and to reduce error factors such as the fluctuations in the intensity of the laser source, phase, or polarization modulation can be used. For this, an electro-optic modulator ${ }^{9}(\mathrm{EOM})$ is added in the optical arrangement between the polarizer and the sensor device. In this case, the output from the photodetector is analyzed for

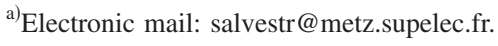

the light intensities of the fundamental and the second harmonic taken as a ratio. The phase shift $\Gamma$ is obtained via the difference between the values of this ratio induced by the physical parameter. ${ }^{9}$ However, the complex signal process reduces the signal to noise ratio in an electronic circuit. Lo and $\mathrm{Hsu}^{10}$ proposed an interesting heterodyne scheme for the demodulation of the optical signal. The principal drawback of this technique is that it requires large modulation depth of the optical signal and thus, large modulation voltage applied on the EOM. Moreover, in the case of this kind of optical sensor, the relationship between the output intensity and the induced phase shift is nonlinear as showed by the transmittance of the polarimetric setup given by the general equation

$$
I=A+B \cos \left[C \cos \left(\omega_{m} t\right)+\Gamma\right],
$$

where $I$ is the output optical intensity, $A$ and $B$ are constants. $C$ is the modulation depth at the modulation frequency $\omega_{m}$, and $\Gamma$ is the phase shift induced by the physical parameter to be measured. To obtain linear transmittance, Garcia-Weidner ${ }^{11}$ proposed a multistage setup in which several sensor devices, with different inclinations of their fast axes, are implemented. In the same way, Yue et al. ${ }^{12}$ used an array of several cascaded parallel Mach-Zehnder interferometers. By adjusting the number of sensor devices, the respective tilt angles, and/or phase shifts of each device, the authors have shown that the linearity of the transmittance could be largely increased. Nevertheless, due to the difficulty of implementation, this scheme cannot found practical application.

To obtain a high linearity of the polarimetric setup response with the phase shift, induced by the physical parameter, the only solution consists in the use of a technique which allows to compensate any induced phase shift. This compensation can be achieved mechanically (polarizer or wave plate rotation), ${ }^{13,14}$ or electrically (electro-optic modulator) as we have proposed in Ref. 13. In this case, the rotation angle or the voltage required for the compensation of the phase shift is proportional to the induced phase shift. This is this approach that we have chosen. The scheme of the corresponding optical sensor is presented in Fig. 1. 


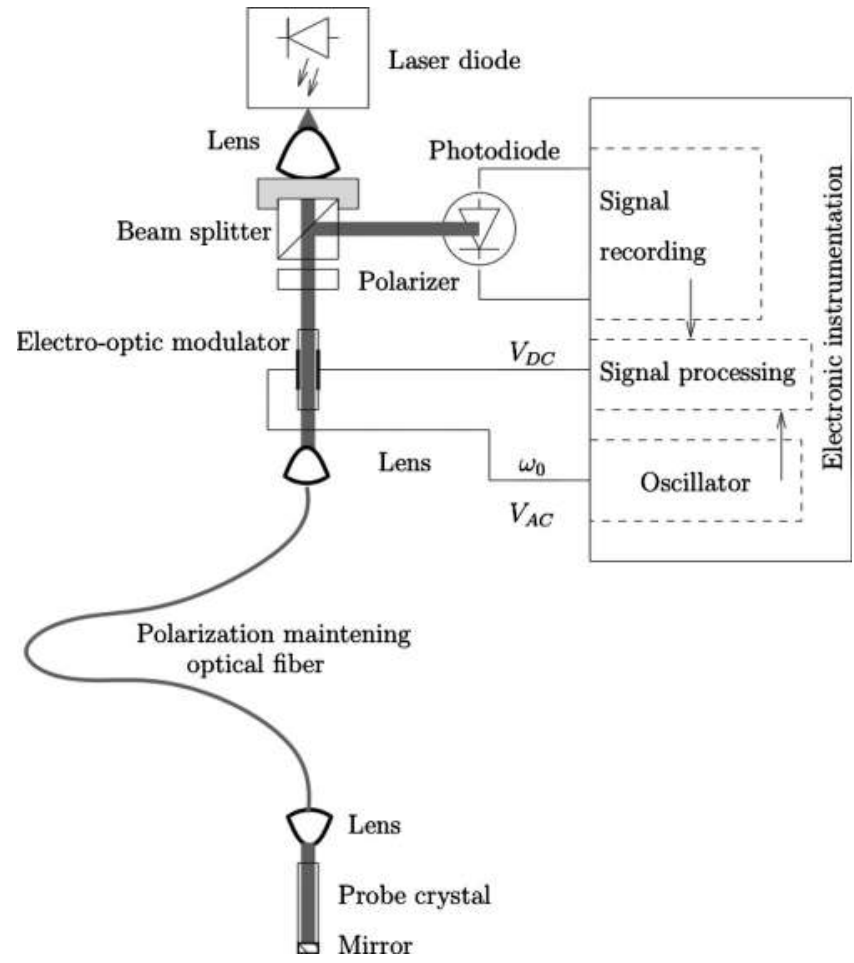

FIG. 1. Proposed optical configuration of the sensor.

To simplify the design of the sensing probe, this optical sensor takes advantage of both bulk optics and optical fiber. Bulk optics is convenient for reducing the required tolerances on optical alignment and also for reducing the sensitivity of the sensor to mechanical vibrations. The optical fibers can transmit light over a long distance with small attenuation and are easily implemented in industrial environments because of their small size, lightweight, immunity to electromagnetic interference, and chemical corrosion resistance. For reason of easier operating and, also to increase the sensitivity (the optical path in two times larger), the optical sensor operates in reflection mode. The EOM is used for both phase modulation of the optical signal and compensation of the induced phase shift. The latter is achieved using a controller based on a closed loop. The output voltage of the controller is proportional to the induced phase shift. In the following, we describe and characterize the operating and the performances of both the optical and electronic parts. As an example, the proposed optical sensor is applied to the measurements of temperature and electric field.

\section{DESCRIPTION OF THE OPTICAL SENSOR AND ITS OPERATING MODE}

To calculate the transmittance of the optical arrangement depicted in the Fig. 1, we have used the Jones formalism. ${ }^{15}$ For the calculus, the arrangement has to be considered in transmission, as described in the Fig. 2. The vibration direction of the linear polarizer $P$ is set to $45^{\circ}$ with respect to both the principal axes $(x, y)$ of the EOM and the crystal probe which are aligned between themselves.

According to the Jones formalism, each optical element can be expressed, with respect to the $(x, y)$ axes system, by the following matrix forms:
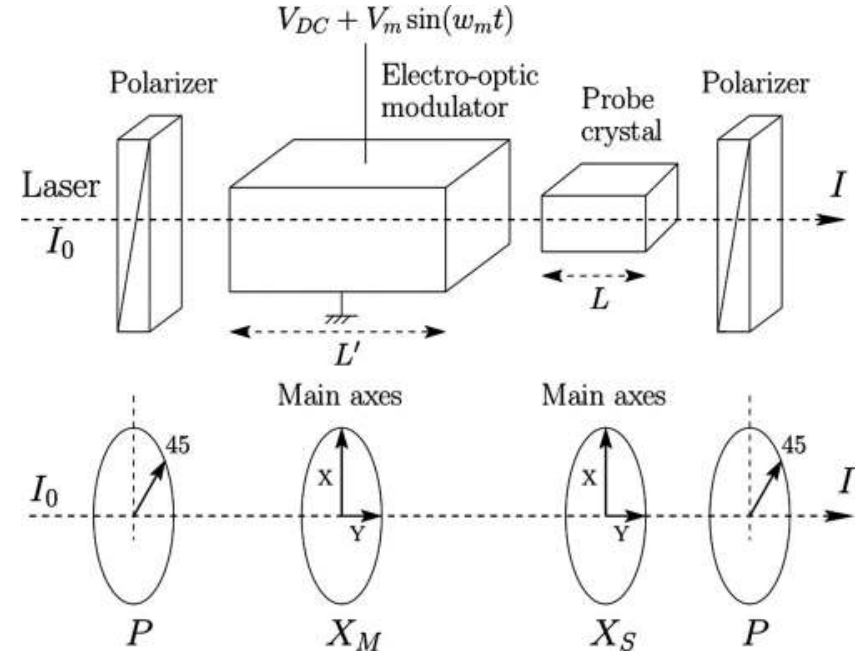

FIG. 2. Scheme of the optical arrangement used for the determination of the optical transmittance showing the orientation of the different optical components along the optical pathway.

$$
P=\frac{1}{2}\left(\begin{array}{ll}
1 & 1 \\
1 & 1
\end{array}\right), \quad X_{M(S)}=\left(\begin{array}{cc}
e^{-l \Gamma^{\prime}(\Gamma) / 2} & 0 \\
0 & e^{-l \Gamma^{\prime}(\Gamma) / 2}
\end{array}\right),
$$

where $\Gamma^{\prime}$ is the phase shift induced by the EOM on which is applied an electrical voltage $V . \Gamma$ is the phase shift induced by the probe crystal via the physical parameter to be measured. The lightwave polarization at the output of the optical system can be thus calculated using

$$
\vec{E}=\left(\begin{array}{c}
E_{x} \\
E_{y}
\end{array}\right)=P X_{S} X_{M} P \frac{1}{2}\left(\begin{array}{l}
1 \\
1
\end{array}\right) \text {. }
$$

After expansion, Eq. (3) becomes

$$
\vec{E}=\frac{1}{2}\left[\begin{array}{c}
\cos \left(\frac{\Gamma+\Gamma^{\prime}}{2}\right) \\
\cos \left(\frac{\Gamma+\Gamma^{\prime}}{2}\right)
\end{array}\right] .
$$

The output intensity is then derived from

$$
I=\frac{E_{x}^{2}+E_{y}^{2}}{2}=I_{0} \cos ^{2}\left(\frac{\Gamma+\Gamma^{\prime}}{2}\right),
$$

where $I_{0}$ is the input optical intensity. Taking into account of the optical losses which can be introduced by reflections and absorptions in the assembly ${ }^{16}$ by the parameter $\gamma=I_{\max }$ $-I_{\min } / I_{\max }+I_{\min }\left(I_{\max }\right.$ and $I_{\min }$ are, respectively, the maximum and minimum values of the output intensity), the transmittance $T=I / I_{0}$ of the arrangement can be written as

$$
T=\frac{1}{2}\left[1+\gamma \cos \left(\Gamma+\Gamma^{\prime}\right)\right] .
$$

In Eq. (6), the phase shifts $\Gamma^{\prime}$ and $\Gamma$, respectively, introduced by the voltage $V=V_{\mathrm{dc}}+V_{m} \sin \left(\omega_{m} t\right)$ applied on the EOM and the probe crystal, can be expressed as

$$
\Gamma^{\prime}=\Gamma_{0}^{\prime}+\frac{\pi\left[V_{\mathrm{dc}}+V_{m} \sin \left(\omega_{m} t\right)\right]}{V_{\pi}}, \quad \Gamma=\Gamma_{0}+\frac{2 \pi L}{\lambda} \delta \Delta n .
$$

$\Gamma_{0}^{\prime}$ and $\Gamma_{0}$ correspond to the phase shifts induced by the natural birefringence of the modulator and the crystal probe, 


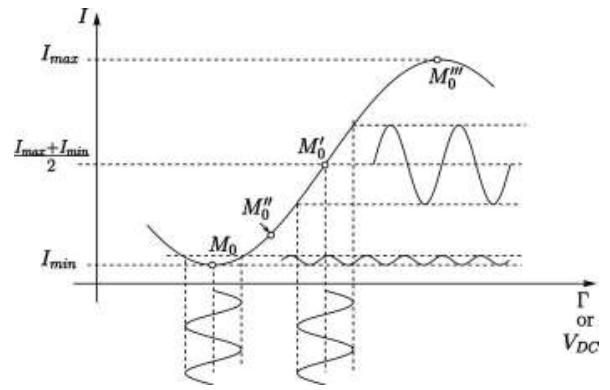

FIG. 3. Transmittance of the setup and the associated operating points.

respectively. $V_{\pi}$ is the half-wave voltage of the modulator. $V_{\mathrm{dc}}$ is the dc voltage allowing the phase shift compensation and $V_{m}$ is the amplitude of the modulating voltage at the pulsation $\omega_{m} . \lambda$ is the wavelength, $L$ is the dimension of the crystal probe along the propagation direction of the lightbeam and $\delta \Delta n$ is the birefringence variation due the physical parameter to be measured. The transmittance curve described by Eq. (6) is presented on Fig. 3.

Replacing Eq. (7) in Eq. (6), and expanding the latter using Bessel function, the transmittance can be written as ${ }^{17}$

$$
T=T_{\mathrm{dc}}+T_{\omega}+T_{2 \omega},
$$

where

$$
\begin{aligned}
& T_{\mathrm{dc}}=\frac{\gamma}{2}\left[1-\left(1-\frac{\Gamma_{m}^{\prime 2}}{4}\right) \cos \left(\Gamma+\Gamma_{0}^{\prime}+\Gamma_{\mathrm{dc}}^{\prime}\right)\right], \\
& T_{\omega}=\frac{\gamma \Gamma_{m}^{\prime}}{2}\left[\left(1-\frac{\Gamma_{m}^{\prime 2}}{8}\right) \sin \left(\omega_{m} t\right) \sin \left(\Gamma+\Gamma_{0}^{\prime}+\Gamma_{\mathrm{dc}}^{\prime}\right)\right], \\
& T_{2 \omega}=\frac{\gamma \Gamma_{m}^{\prime 2}}{8}\left[\left(1-\frac{\Gamma_{m}^{\prime 2}}{12}\right) \cos \left(2 \omega_{m} t\right) \cos \left(\Gamma+\Gamma_{0}^{\prime}+\Gamma_{\mathrm{dc}}^{\prime}\right)\right],
\end{aligned}
$$

$\Gamma_{\mathrm{dc}}^{\prime}$ and $\Gamma_{m}^{\prime}$ are given by

$$
\Gamma_{\mathrm{dc}}^{\prime}=\frac{\pi\left[V_{\mathrm{dc}}\right]}{V_{\pi}}, \quad \Gamma_{m}^{\prime}=\frac{\pi\left[V_{m} \sin \left(\omega_{m} t\right)\right]}{V_{\pi}} .
$$

Equation (8) describes the intensity of light which is detected by the system at any instance in time. As it can be seen in Eq. (9), the detected signal consists of a dc term, a frequency-doubled term, and the signal of interest at the modulation frequency $\omega_{m}$ which is used, as we will see below, as the input into the control system. Two typical cases have to be considered. In one hand, if the voltage $V_{\mathrm{dc}}$ applied to the modulator is such that $\Gamma+\Gamma_{0}^{\prime}+\Gamma_{\mathrm{dc}}^{\prime}= \pm k \pi, k$ $=0,1,2, \ldots$, then the system is set at the operating points $M_{0}$ with

$$
T_{\mathrm{dc}}=T_{\omega}=0, \quad T_{2 \omega}=\frac{\gamma}{2} \frac{\Gamma_{m}^{\prime 2}}{4}\left[\left(1-\frac{\Gamma_{m}^{\prime 2}}{12}\right) \cos \left(2 \omega_{m} t\right)\right]
$$

for even values of $k$, and $M_{0}^{\prime \prime \prime}$ :
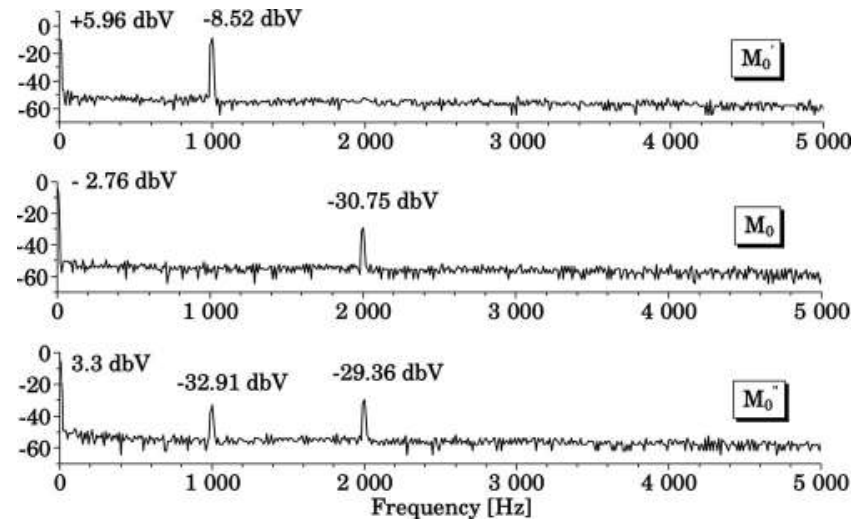

FIG. 4. Fourier transform of the output optical signal for a modulation frequency of $1 \mathrm{kHz}$, and recorded at different operating points: (top) at the $M_{0}^{\prime}$ point (linear zone of the transmittance); (middle) at $M_{0}$ or $M_{0}^{\prime \prime \prime}$; (bottom) at $M_{0}^{\prime \prime}$ (between $M_{0}$ and $M_{0}^{\prime}$ ).

$$
T_{\mathrm{dc}}=\gamma, \quad T_{\omega}=0, \quad T_{2 \omega}=\frac{\gamma}{2} \frac{\Gamma_{m}^{\prime 2}}{4}\left[\left(1-\frac{\Gamma_{m}^{\prime 2}}{12}\right) \cos \left(2 \omega_{m} t\right)\right]
$$

for odd values of $k$. In the other hand, if the voltage $V_{\mathrm{dc}}$ applied to the modulator is such that $\Gamma+\Gamma_{0}^{\prime}+\Gamma_{\mathrm{dc}}^{\prime}=\pi / 2 \pm k \pi$, $k=0,1,2, \ldots$, then the system is set at the operating points $M_{0}^{\prime}$ with

$$
T_{\mathrm{dc}}=\frac{\gamma}{2}, \quad T_{\omega}= \pm \frac{\gamma}{2} \Gamma_{m}^{\prime}\left[\left(1-\frac{\Gamma_{m}^{\prime 2}}{8}\right) \sin \left(\omega_{m} t\right)\right], \quad T_{2 \omega}=0
$$

The output signals as obtained in these different operating points are shown in Fig. 4.

For this, we have used the optical sensor as depicted in Fig. 1, but without the optical fiber and with a HeNe laser $(\lambda=633 \mathrm{~nm}, P=2 \mathrm{~mW})$ as a source. The EOM is based on a lithium niobate crystal for which the light propagates along the optical axis (z-axis, $40 \mathrm{~mm}$ long) and the voltage is applied along the $y$-axis ( $3 \mathrm{~mm}$ in thickness). In this configuration the value of $V_{\pi}$ is equal to $190 \mathrm{~V}$. This rather large voltage is due to the low value of the electro-optic coefficient involved in this configuration $\left[\mathrm{r}_{22}=6.4 \mathrm{pm} / \mathrm{V}\right.$ at $\lambda$ $=632.8 \mathrm{~nm}$ (Ref. 18)] which offers the large advantage of a temperature-insensitivity operating of the modulator, since the natural birefringence is null. The different operating points were adjusted via the voltage $V_{\mathrm{dc}}$ applied on the EOM.

\section{CONTROL SYSTEM}

The best sensitivity for the detection of any shift of the operating point is obtained when the latter is set to $M_{0}$, and by measuring the signal amplitude at the fundamental frequency. Indeed, as it can be seen on Fig. 4, a shift of the operating point from $M_{0}$ to $M_{0}^{\prime \prime}$ induces a variation of the signal amplitude at the fundamental frequency of more than $32 \mathrm{~dB} \mathrm{~V}$, while the signal amplitude at the first harmonic frequency experiences only $1 \mathrm{~dB} \mathrm{~V}$ of amplitude variation.

The purpose of the control system is thus to maintain the system at the operating point $M_{0}$, by adjusting the voltage 


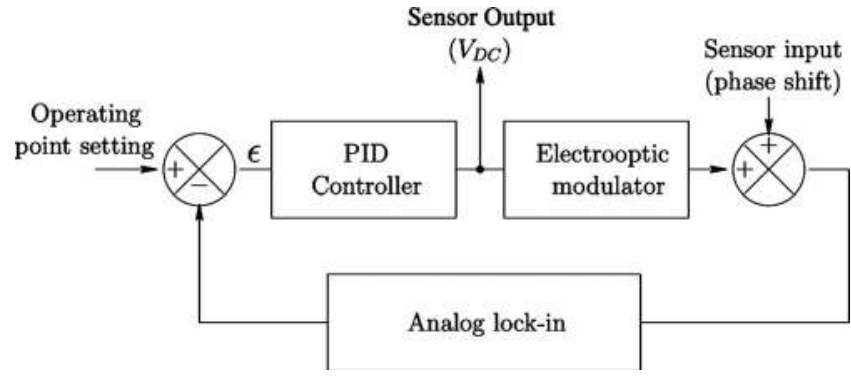

FIG. 5. Block diagram of the designed and implemented analog closed-loop controller.

$V_{\mathrm{dc}}$ applied to the modulator, in order to compensate for any phase shift $\Gamma$ due to the crystal probe. Then the condition $\Gamma+\Gamma_{0}^{\prime}+\Gamma_{\mathrm{dc}}^{\prime}= \pm k \pi, k=0,1,2, \ldots$ yields

$$
\Delta n= \pm \frac{\lambda}{2 V_{\pi} L}\left(k V_{\pi}-1\right) V_{\mathrm{dc}} .
$$

After compensation, the output voltage of the controller is proportional to the voltage $V_{\mathrm{dc}}$ applied on the EOM and thus to the birefringence variation induced by the crystal probe. The knowledge of the relationship between the birefringence variation and the physical quantity to be measured allows its determination. In order to achieve this, an analog proportional, integral, derative (PID) control system was implemented. The general block diagram of the control system is illustrated in Fig. 5. The input into the system control is the phase shift due to the birefringence change induced by the physical parameter to be measured. The purpose of the analog lock-in is to measure the amplitude of the sinusoidal component of the detected signal at the modulation frequency $\omega_{m}$. The output of the lock-in, as well as the operating point setting voltage, are then sent into a voltage comparator. The output of the comparator is then transmitted as an input to a PID controller which adjusts the voltage applied on the modulator in order to minimize the difference between the two voltages, and thus, to keep the operating point at the same position.

Figure 6 shows a schematic view of both electronic and

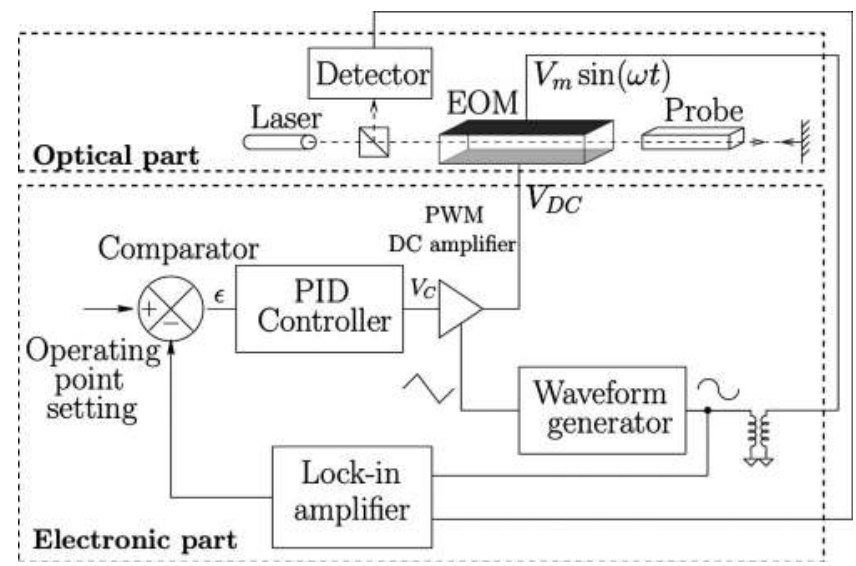

FIG. 6. Schematic of both electronic and optical instrumentations of the sensor. optical instrumentations of the sensor. In the following, we give more details on the realization of the different electronic parts.

\section{A. Modulation and dc voltages}

To obtain the modulation voltage, we have used the sinusoidal output of a XR2206 (EXAR Industries) waveform generator integrated circuit (IC), cascaded with a transformer allowing to increase the voltage delivered by the IC by one order of magnitude. We finally get a modulation voltage rms value adjustable up to $52 \mathrm{~V}$ at a frequency of $20.7 \mathrm{KHz}$ with very low distortion and small frequency and amplitude drifts. The dc voltage is obtained using a CA05P (EMCO) dc-dc converter IC as a high voltage source (maximum rms value of $740 \mathrm{~V}$ ), coupled with a pulse-width modulation (PWM) circuit allowing a linear variation of the dc output voltage between 80 and $740 \mathrm{~V}$ for driving voltage varying between -3 and $+3 \mathrm{~V}$. The rise and fall times are equal to $20 \mathrm{~ms}$. The modulation signal for the PWM circuit which is implemented with an AD620 (Analog Devices) voltage comparator IC and a BU505 (Philips) transistor, comes from the triangular output voltage of the XR2206 IC.

\section{B. Optical detector and lock-in amplifier}

The optical signal is converted to a voltage signal by a small surface silicon photodiode (gain $=0.1 \mathrm{~A} / \mathrm{W}$ ) cascaded with a current-to-voltage amplifier based on an AD844 IC. The corresponding output signal is then filtered, using a selective bandpass filter centered at $20.7 \mathrm{kHz}$, and multiplied with the reference signal delivered by XR2206 IC, using an AD633 multiplier IC. This signal multiplication results in a frequency shift of the modulated signal from $20.7 \mathrm{kHz}$ down to a null frequency. The output of the multiplier is then amplified and filtered with a first order lowpass filter $\left(f_{c}\right.$ $=300 \mathrm{~Hz}$ ). The latter dc voltage is thus proportional to the amplitude of the signal at the modulation frequency $\omega_{m}$ $=20.7 \mathrm{kHz}$, and allows its measurement.

\section{PID controller}

The PID controller is built using a parallel structure combining the three basic operations. Each operation is implemented using operational amplifier based classical electronic circuits. In order to calculate the PID controller's parameters, which are the control gain $K$, and integration and derivative times $T_{I}$ and $T_{D}$, respectively, the system was studied in open-loop using the Ziegler-Nichols method. ${ }^{19}$ First, as shown in Fig. 7, to illustrate the operating of the system in open loop, we have recorded the evolution of the output voltage $V_{S D}$ of the lock-in amplifier with respect to the voltage $V_{\mathrm{dc}}$ applied on the modulator. Around the operating point $M_{0}$, in the range $-40 \leq V_{\mathrm{dc}} \leq 0$, the amplitude $V_{S D}$ of the modulated signal at the frequency $\omega_{m}$ is varying linearly with the voltage $V_{\mathrm{dc}}$. As expected, on each side of the transmittance curve with respect to $M_{0}$, the sign of $V_{S D}$ is opposite, and thus, leads to the determination of the sign of the physical parameter variation. It is to be noticed, that the voltage $V_{S D}$ corresponding to the operating point $M_{0}$, is not exactly 


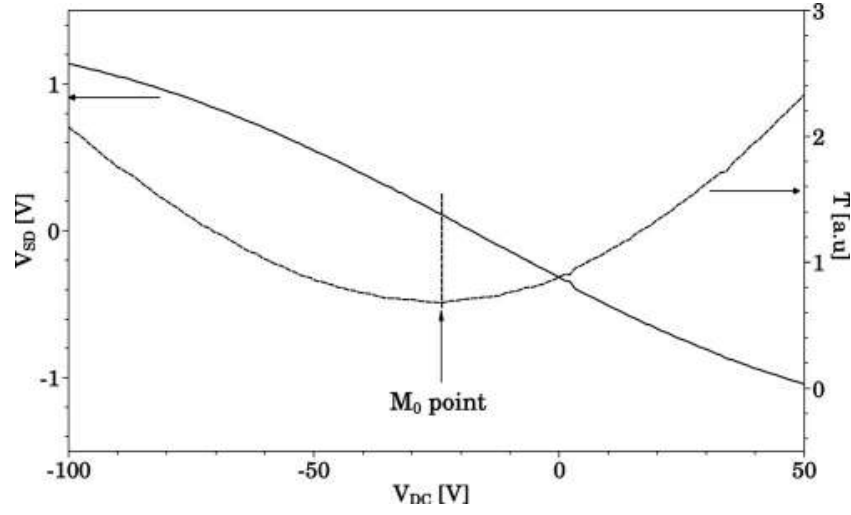

FIG. 7. (a) Evolution of the output voltage $V_{S D}$ of the lock-in amplifier with respect to the voltage $V_{\mathrm{dc}}$ applied on the modulator. (b) Corresponding transmittance curve $T$ as recorded at the output of the detector.

null. This is due to residual offsets of the integrated circuits which can be easily adjusted to the desired value.

The Ziegler-Nichols method relies on the analysis of the time response (see Fig. 8) of the output voltage $V_{S D}$ (in open loop) to a voltage step of $V_{\mathrm{dc}}$. This analysis, which consists in the measurement of both the slope of the rising response and the delay time of this response with respect to the voltage step input voltage, leads to the PID controller's values which were found to be equal to $K=75, T_{i}=0.25 \mathrm{~ms}$, and $T_{d}=0.125 \mathrm{~ms}$.

\section{PERFORMANCES OF THE OPTICAL SENSOR}

\section{A. Stability}

From a general point of view, the stability of operating is a key parameter for sensors. It is thus important to check that the output of the implemented sensor does not drift with time after the input sensor compensation. For this, the sensor was studied, at room temperature, without the probe crystal, and the operating point was set to $M_{0}$. A voltage step $\Delta V_{\mathrm{dc}}$ (simulating the sensor input) was applied on the EOM. The voltage compensation $V_{\mathrm{dc}}$, was recorded during $8 \mathrm{~h}$ (see Fig. 9).

Under these conditions, and once the steady state of the compensation voltage is reached, any drift of the output should be observed. The analysis of the results plotted in Fig. 9 reveals a quite weak variation of the output of the sensor, which can be estimated to be less than $3 \%$ over $8 \mathrm{~h}$. This

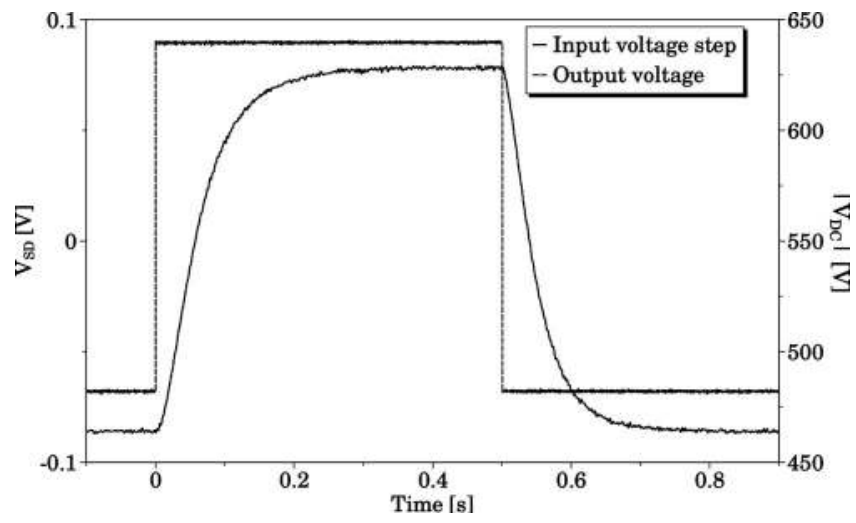

FIG. 8. Time response of the output voltage $V_{S D}$ (in open loop) to a voltage step of $V_{\mathrm{dc}}$.

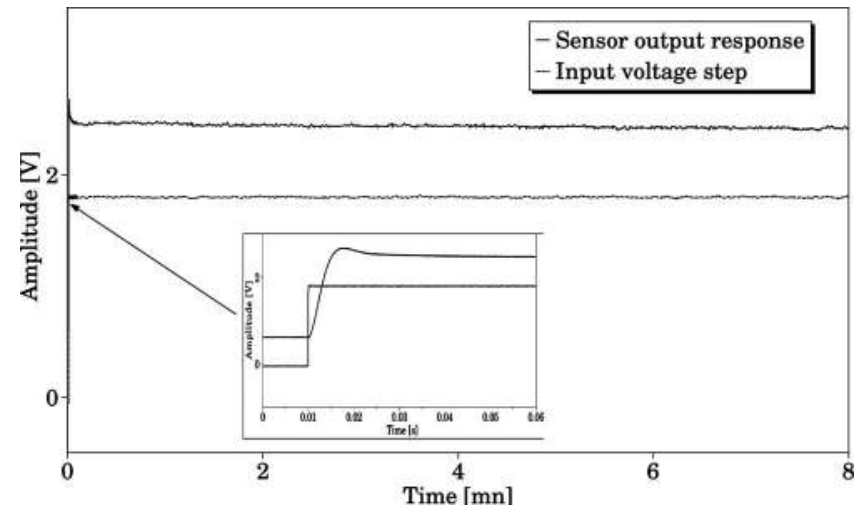

FIG. 9. Time dependence of the voltage compensation $V_{\mathrm{dc}}$ (without crystal probe).

variation could originate from the temperature drift of the electronic components (IC, resistors, capacitance, etc.), from residual temperature drift of the EOM, and from possible laser source fluctuations. To check the latter point, as it is shown in Fig. 10, we have recorded the sensor output voltage, while the laser source was submitted to random intensity variations. As it can be seen, even for very large variation of the laser intensity, the sensor output remains very stable, and the variations which are observed are not correlated with those of the laser intensity.

This is due to the choice of the operating point $M_{0}$ of the sensor. The analysis of the transmittance curve, given by Eq. (6), for different values of the input intensity $I_{0}$, and represented on Fig. 11, shows that, at this particular point, and contrarily to any other operating point, the increase or decrease in the input intensity does not change the mean value of the intensity, and thus, does not affect the amplitude of the modulated signal on which is based the control system.

\section{B. Response time}

In order to measure the response time of the optical sensor, the output voltage of the PID controller, namely, the error signal, as well as the voltage step $\Delta V_{\mathrm{dc}}$ simulating the sensor input, were recorded simultaneously, as it is shown on Fig. 12. As it can be seen, the error voltage first increases from $0 \mathrm{~V}$ up to its maximum value in $10 \mathrm{~ms}$, and then decreases to reach its initial null value, indicating a full

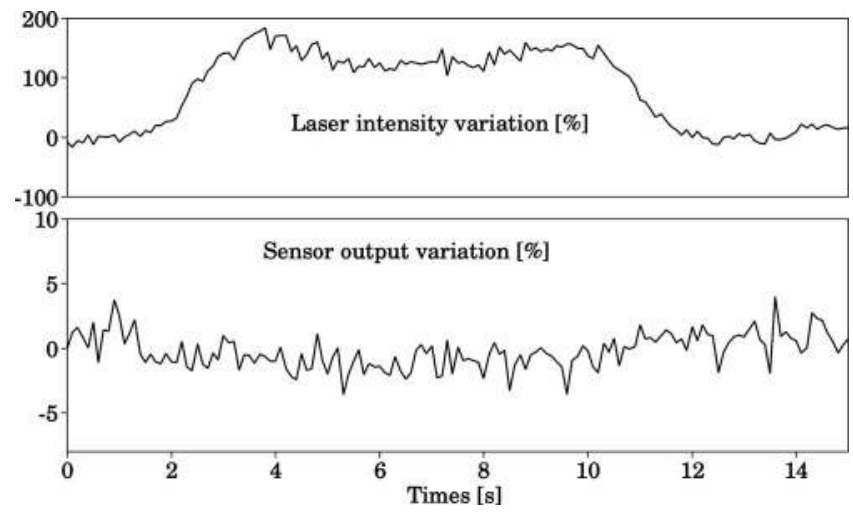

FIG. 10. Record of the sensor output voltage variations in the case of laser source intensity variations. 


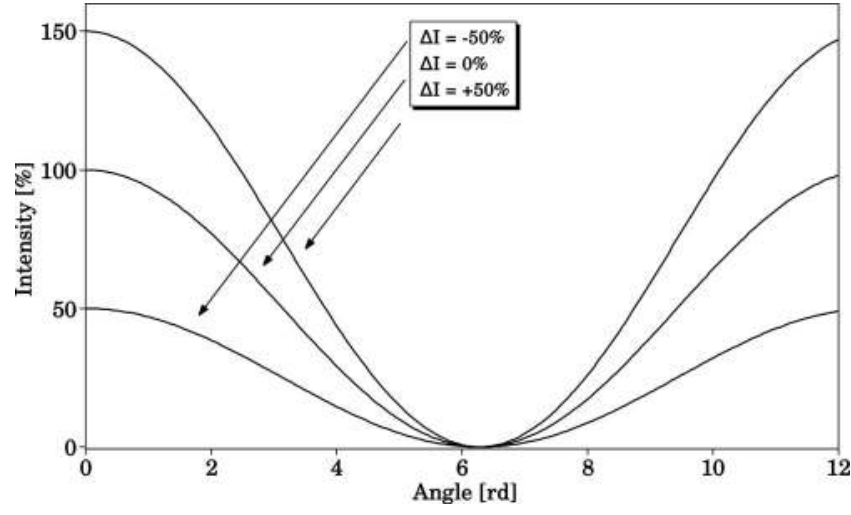

FIG. 11. Transmittance curves for different values of the input intensity $I_{0}$. The mean intensity at the operating point $M_{0}$ is not affected by laser intensity fluctuations.

compensation, in $80 \mathrm{~ms}$. The response time that can be deduced from this measurement is found to be equal to $50 \mathrm{~ms}$. This value, corresponding to a closed-loop operating, is, as expected, lower than those of the system operating in open loop, that is deduced from the measurement plotted in Fig. 8, which is equal to $200 \mathrm{~ms}$.

\section{Optical sensor in temperature and electric field measurements modes}

We have used the implemented optical sensor for the measurement of temperature and electric field variations. The birefringence shift induced by temperature and electric field variations $d T$ and $d E$, can be expressed as

$$
d \Delta n= \pm \frac{\lambda}{2 T_{\pi}\left(\mathbf{E}_{\pi}\right) L^{\prime}} d T(\mathbf{d E}) .
$$

$L^{\prime}$ is the length of the crystal probe along the light propagation direction. $T_{\pi}$ and $E_{\pi}$ are the typical values of the temperature and electric field, that introduced a phase shift of $\pi$, given by

$$
T_{\pi}\left(\mathbf{E}_{\pi}\right)=\frac{\lambda}{2 L^{\prime} \alpha_{\text {eff }}\left(\boldsymbol{n}_{\text {eff }}^{3} r_{\text {eff }}\right)},
$$

where $n_{\text {eff }}, \alpha_{\text {eff }}$, and $r_{\text {eff }}$ are the effective refractive index, thermo-optic, and electro-optic coefficients, respectively, of the crystal probe. According to Eqs. (14) and (15), we can

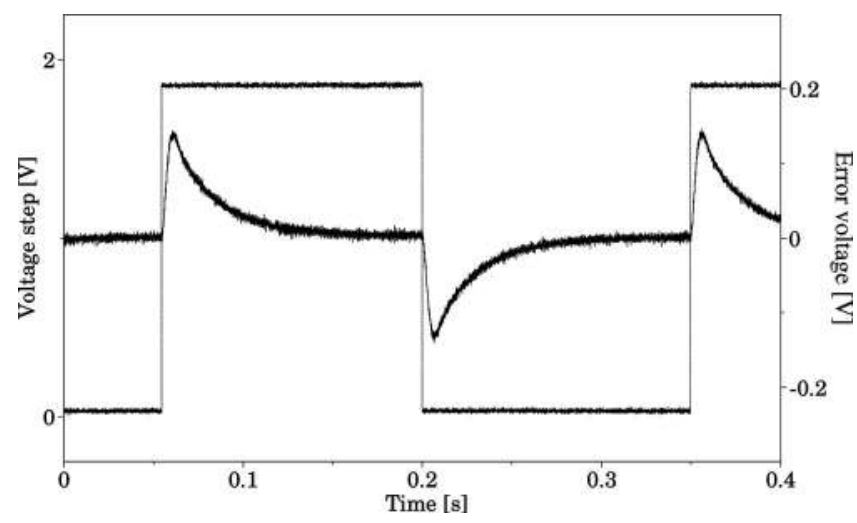

FIG. 12. Recorded PID controller output voltage (error voltage) when a voltage step $\Delta V_{\mathrm{dc}}$ (simulating the sensor input) is applied on the EOM.

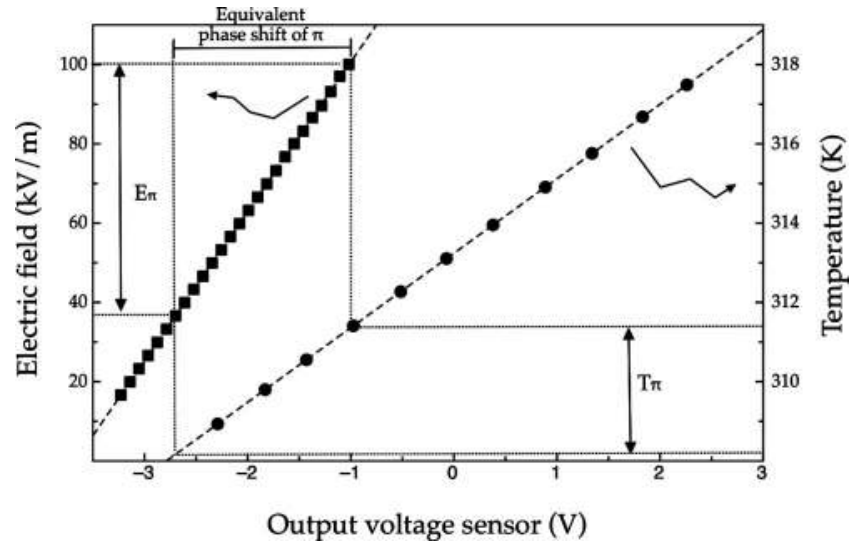

FIG. 13. Record of the sensor output voltage for both temperature and electric field variation measurements.

thus derive the relative variations of temperature and electric field measured by the sensor, via the compensation voltage $V_{\mathrm{dc}}$, as

$$
\frac{d T}{T_{\pi}} \quad \text { or } \quad \frac{d E}{E_{\pi}}= \pm \frac{L^{\prime}}{L}\left(k V_{\pi}-1\right) \frac{V_{\mathrm{dc}}}{V_{\pi}} .
$$

Equation (17) shows that the largest sensitivity is obtained for crystal probes possessing, as expected, large optical interaction length $\left(L^{\prime}\right)$, low values of $T_{\pi}$ and $V_{\pi}$, and thus for crystals exhibiting large thermo-optic and electro-optic coefficients, for temperature and electric field sensing, respectively. For electric field sensing, we have chosen as crystal probe, a $\mathrm{LiNbO}_{3}$ crystal with the same dimension and configuration than those of the crystal used to build the EO modulator. This choice was made for two reasons. First, within this configuration, the crystal probe is not sensitive to temperature since the natural birefringence is null for light propagation along the $z$-optical axis. Second, since both crystal dimensions are equal, the compensation voltage $V_{\mathrm{dc}}$ should be exactly equal to those applied on the crystal probe and, thus, the good operating of the sensor can be easily checked. For temperature sensing, as an example, we have chosen a $\mathrm{KTiOP}_{4}$ crystal with $l \times d \times L=3 \times 3 \times 10 \mathrm{~mm}^{3}$ in dimension as crystal probe. $l, d$, and $L$ are, respectively, the lengths of the crystal probes along the $y, z$, and $x$ axes. In this configuration, the value of the thermo-optic coefficient is rather low $\left[\alpha_{\text {eff }}=5 \times 10^{-6} \mathrm{~K}^{-1}\right.$ (Ref. 20)] leading to a value of $T_{\pi}=3.16 \mathrm{~K}$ and thus, to a rather low sensitivity.

The results obtained for temperature and electric field variations measurements are reported in Fig. 13. This figure

TABLE I. Summary of the performances of the implemented optical sensor in temperature and electric field measurement mode.

\begin{tabular}{lcc}
\hline \hline Parameter & Temperature measurements & Electric-field measurements \\
\hline Sensitivity & $0.53 \mathrm{~V} / \mathrm{K}$ & $27 \mu \mathrm{V} / \mathrm{V} \mathrm{m}^{-1}$ \\
\hline Resolution & $7.5 \mathrm{mK}$ & $148 \mathrm{~V} \mathrm{~m}^{-1}$ \\
Accuracy & $\pm 9.5 \mathrm{mK}$ & $\pm 185 \mathrm{~V} \mathrm{~m}^{-1}$ \\
Response time & $50 \mathrm{~ms}$ & $50 \mathrm{~ms}$ \\
Linearity error & $\pm 2.2 \%$ & $\pm 2.2 \%$ \\
Gain error & $\pm 1.7 \%$ & $\pm 1.7 \%$ \\
\hline \hline
\end{tabular}




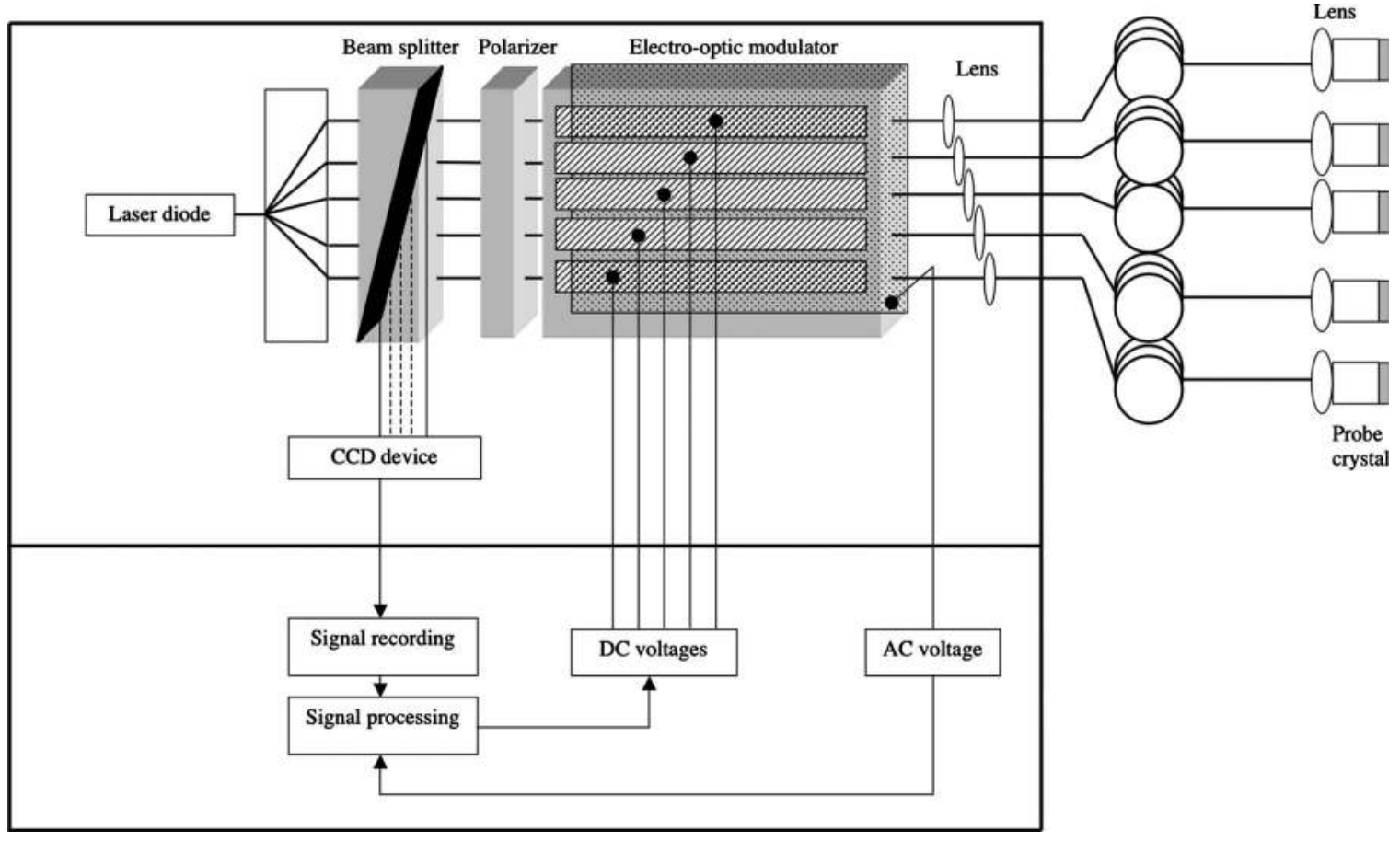

FIG. 14. Schematic of a possible multiprobe crystals sensor.

shows an excellent linear behavior (linearity error of $2.2 \%$ and gain error of $1.7 \%$ ) of the sensor output. The values of the sensitivity of the optical sensor in both temperature and electric field measurements mode can be deduced from the slopes of the two curves and are reported in Table I. These two curves allow to infer roughly the values of both $T_{\pi}$ and $E_{\pi}$, since a phase shift of $\pi$ corresponds to a variation of the output voltage $V_{\mathrm{dc}}$ equal to $1.72 \mathrm{~V}$ (see Fig. 13). These values were found to be in a very good agreement with those calculated using Eq. (16).

Two other parameters are of importance for a sensor. From one hand, the accuracy of the optical sensor can be deduced from a statistical study of several hundred measurements of both temperature and electric field variations. In the other hand, the maximum level of noise of the output voltage can give the resolution of the sensor. All these values have been calculated and are reported in Table I. It is to be noticed that the choice of the crystal probes (material and dimensions) was not optimized. Materials with thermo-optic coefficient larger by one $\left[\mathrm{LiNbO}_{3},{ }^{21} \mathrm{LiTaO}_{3}\right.$ (Ref. 22)] or two order of magnitude $\left[\mathrm{CdGeP}_{2}\right.$ (Ref. 23)] are obviously a better alternative than $\mathrm{KTiOPO}_{4}$ (KTP) crystal. For instance, the use of $\mathrm{CdGeP}_{2}$ as material for the crystal probe would allow to reduce the length of the crystal down to $3 \mathrm{~mm}$ and increase the temperature resolution up to less than $1 \mathrm{mK}$. In the same way, for electric field sensing, the resolution could be increase up to $0.05 \mathrm{~V} / \mathrm{m}$ with the use of a $1 \mathrm{~mm}^{3}$ PLZT (Ref. 24) crystal probe. The total error in the response of this optical sensor is equal to less than $4 \%$ and its resolution in term of phase shift is equal to $\pm 23.0 \mathrm{mrad}$. The rather large response time is mainly originating from the PWM amplifier, and can be easily reduced to less than $1 \mathrm{~ms}$ by increasing the modulation frequency of the PWM amplifier by one order of magnitude.

\section{v. CONCLUSION}

We have developed new compact, linear, highly sensitive, and versatile polarimetric sensor based on the use of an EO phase modulator. This optical sensor relies on a measurement technique which is not dependent on the crystal probe used, and thus can be adapted and optimized, by a convenient choice of the material, to probe different physical properties. Attention is currently paid to the electronic part of the sensor to increase the performances, in terms of accuracy, resolution, and response time. We also are working to expand the dynamic range of the sensor, which is limited by the EOM applied dc voltage range and actually equal to $3.5 \mathrm{~V}_{\pi}$. For this, a full digital control of the optical sensor, via a microcontroller, will be implemented. It will allow to set the dc voltage, when the saturation value is reached, to its initial value without any information loss.

At longer term, the system will be modified to operate in multiprobe crystals mode, in order to sense simultaneously, as it is shown in Fig. 14, several and/or different physical properties. As an example of application, the proposed sensor, when embedded in composite laminates could be thus used to detect the development of the matrix cracking. It could be also used for the instrumentation in nuclear power plants. Among the physical parameters needed to be determined in this case, temperature, pressure, strain, electromagnetic field, radiation doses, and gas concentrations are the most prevalently quoted.

${ }^{1}$ E. Udd, Fiber Optic Sensors: An introduction for engineers and scientists (Wiley, New York, 1991).

${ }^{2}$ S. M. Jeon and Y. P. Kim, Opt. Laser Technol. 36, 181 (2004).

${ }^{3}$ W. K. Kuo, W. H. Chen, Y. T. Huang, and S. L. Huang, Appl. Opt. 39, 4985 (2000)

${ }^{4}$ F. Cecelja, M. Bordovsky, and W. Balachadran, IEEE Trans. Instrum. Meas. 51, 282 (2002). 
${ }^{5}$ A. Kumada, M. Chiba, and K. Hidaka, J. Appl. Phys. 84, 3059 (1998).

${ }^{6}$ J. R. Mackey, K. K. Das, S. L. Anna, and G. H. McKinley, Meas. Sci. Technol. 10, 946 (1999).

${ }^{7}$ W. Lukosz, Ch. Stamm, H. R. Moser, R. Ryf, and J. Dübendorfer, Sens. Actuators B 39, 316 (1997).

${ }^{8}$ B. D. Cameron and G. L. Cote, IEEE Trans. Biomed. Eng. 44, 1221 (1997).

${ }^{9}$ Y. Shindo and H. Hanabusa, Polym. Commun. 24, 240 (1983).

${ }^{10}$ Y.-L. Lo and P.-F. Hsu, Opt. Eng. (Bellingham) 41, 2764 (2002).

${ }^{11}$ A. Garcia-Weidner, Appl. Opt. 32, 7313 (1993).

${ }^{12}$ Z. Yue, S. Tang, and R. T. Chen, Proc. SPIE 3288, 232 (1998).

${ }^{13}$ M. Aillerie, P. Kolata, J. P. Salvestrini, and M. Fontana, French Patent No. 9609271 (19 July 1997).

${ }^{14}$ M. Bernier, G. Gaborit, L. Duvillaret, A. Paupert, and J.-L. Lasserre, Appl. Opt. 47, 2470 (2008).

${ }^{15}$ R. C. Jones, J. Opt. Soc. Am. 31, 488 (1941); P. S. Theocaris and E. E. Gdoutos, Matrix Theory of Photoelasticity (Springer, Berlin, 1979).
${ }^{16}$ M. Aillerie, F. Abdi, N. Théofanous, M. D. Fontana, and E. Abarkan, Rev. Sci. Instrum. 71, 1627 (2000).

${ }^{17}$ N. Theophanous, M. A. Ahmed, and G. Alexakis, Opt. Quantum Electron. 21, 363 (1989).

${ }^{18}$ M. Abarkan, J. P. Salvestrini, M. D. Fontana, and M. Aillerie, Appl. Phys. B: Lasers Opt. 76, 765 (2003).

${ }^{19}$ J. G. Ziegler and N. B. Nichols, Trans. ASME 64, 759 (1942).

${ }^{20}$ V. G. Dmitriev, G. G. Gurzadyan, and D. N. Nikogosyan, Handbook of Nonlinear Optical Crystals (Springer, New York, 1993).

${ }^{21}$ G. D. Boyd, W. L. Bond, and H. L. Carter, J. Appl. Phys. 38, 1941 (1967).

${ }^{22}$ K. S. Abedin and H. Ito, J. Appl. Phys. 80, 6561 (1996).

${ }^{23}$ G. D. Boyd, E. Buehler, F. G. Storz, and J. H. Wernick, IEEE J. Quantum Electron. 8, 419 (1972).

${ }^{24}$ A. S. Vasilevskaya, I. M. Grodnenskii, A. S. Levichev, I. A. Slepkov, A. V. Smolya, A. S. Sonin, and N. B. Fel'dman Bull. Acad. Sci. USSR, Phys. Ser. (Engl. Transl.) 39, 148 (1975). 